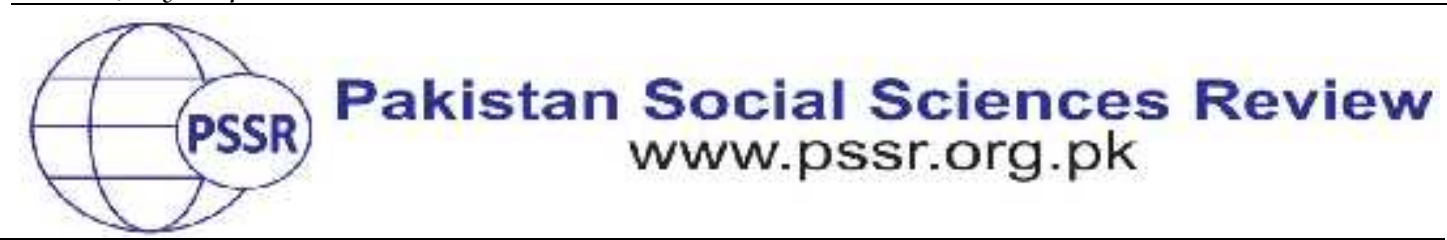

RESEARCH PAPER

\title{
A Corpus Analysis of Online Education Tweets During Covid-19
}

\author{
Dr. Urooj Fatima Alvi ${ }^{1}$ Dr. Shafqat Rasool ${ }^{2}$
}

1. Assistant Professor, Department of English Department, University of Education, Lahore, Punjab, Pakistan

2. Professor, Department of Education, Govt.. College University Faisalabad, Punjab, Pakistan

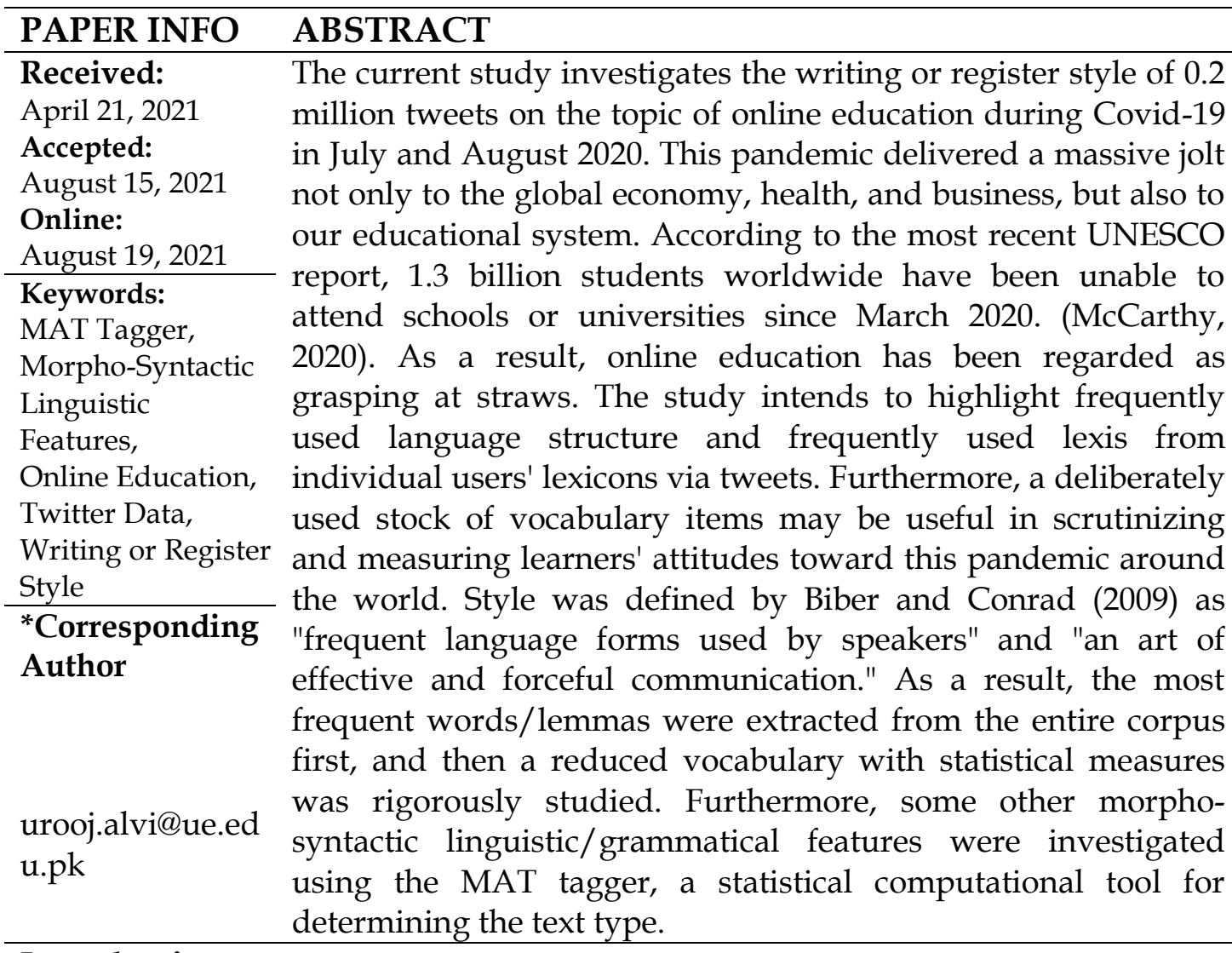

\section{Introduction}

Burghardt (2015) refers to the striking importance of social media language data as it is user-generated that leads to linguistic exploration, for example, its vocabulary, style, grammar, and meaning. Academically, the study of Twitter data has become an immensely popular field of interest. Rogers (2013) has provided prominent academic research on Twitter. Researchers have explored the topics such as information behavior in Twitter, sentiment analysis, identification of parts of speech (POS) tagging, linguistic redundancy in Twitter language, identification of the linguistic structure of sarcasm, analyzing out of useful vocabulary words, 
classification of text linguistics, and numerous other language issues remained as a major influence. This paper also supports the same growing field of interest. It is a corpus analysis of the largest set of tweets on the hot topic of 'online education. It thoroughly examines individuals' linguistic expression or register analysis of the international tweeter community. It applies the latest corpus linguistic tool of MAT tagger that is used for multidimensional analysis of any language data. The tagger is a statistical and computational tool that automatically runs on the data and as a result, provides the functional dimensions of the text. These functional dimensions determine the writing/text style by tagging and automatically counting the cooccurrence of sets of linguistic features. As a result, comprehensive grammatical information of linguistic features and their values of descriptive statistics (mean, median, mode, and standard deviation) are available. Based on these values, along with the language behaviors, frequently used vocabulary items are documented.

Register means language use along with the function of language or in simple words specific vocabulary usage according to social occasion, purpose, audience, and context. The tagger has the means values of all text types according to the popular framework of Biber (1988).

\section{Literature Review}

Anguilera-Hermida (2020) conducted research that was based on the acceptance of online learning during the emergency times of Covid-19. While exploring the students' perceptions in this study regarding the entirely new higher education system, it analyzed two fundamental points. Firstly, the psychological factors impact strongly on students' motivation, attitude, self-efficacy, affect, and cognitive engagement. Secondly, it also impacts students' willingness to learn. It was surveyed among 270 learners. It resulted in a great fluctuation in students' learning behaviors during emergency times.

In the same way, Oyedotun (2020) designed a study in terms of online education considering the developing country of Guyana. The focus of the research was to bring out the sudden change and its overall impact. It was concluded that online education proved a challenge. It has disrupted the local education sector.

Another survey-based research, conducted by Mishra, Gupta, and Shree (2020) examined online teaching learning in higher education withinthe Indian context. The research was conducted to address the required measurement and transformation of formal education into online education. It was inferred that to ensure the resumption of quality educational activities, it was obligatory to establish the linkage between change management as a normal course of the procedure.

Clain, Gibbs \& McRae (2020) focused on a research study that all students or learners should get equal opportunities in learning and education. According to the concept of inclusive education, regardless of differences in social, cultural, 
emotional, intellectual, linguistic, or any other, learners should attend their school of choice. They found that news media should be used to echo this voice of equality.

Abbasi, et al (2020) conducted a descriptive and cross-sectional study to determine that how would students perceive e-learning during the lockdown. After receiving 382 responses, it was observed that $77 \%$ of students had extremely negative perceptions towards, e-learning. Moreover, $76 \%$ of students used their mobile devices for the purpose of e-learning. Even in lockdown situations, learners avoided e-teaching against face-to-face teaching. It was concluded in the study that the faculty members and administrations must take steps to improve e-teaching pedagogy during the time of crises.

Slimi (2020) also reported challenges in online teaching in higher education under confinement circumstances. The study also identified the opportunities in the new system coupled with providing the solutions in the problematic area. In 64 responses, it was observed that besides a novel experience of online teaching, a considerable number of learners have enjoyed the change. It was considered an independent state of learning various skills for example problem-solving communication, IT and technological skills, etc. However, the hurdles were counted as poor internet connectivity and missing readiness for academic activities.

Shehzad, et al, 2020 contributed to observe the impact of virtual teaching in Pakistani post-graduate students concerning ESL learners. It was found that virtual teaching during the Covid-19, enormously affected academic staff, learners, and institutes in terms of learning, time, and finance. Although students showed an optimistic attitude for this challenge.

Bao (2020) investigated the online teaching situation in one of the famous universities of China, i.e., a case study on Peking University. By focusing on five instructional strategies, the study inferred that firstly students' anxiety must be addressed, secondly, there must be contingency plans for resolving traffic overload issues while online teaching and lastly, there must be timely feedback, email guidance, and high-quality participation. In this study, six specific instructional strategies were based on improved instructional design, effective delivery, adequate support, massive participation, and contingency plans to deal with unexpected incidents. Overall, it was a situational analysis of the shift between face-to-face education to online that demanded a lot of improvement.

After the overall pandemic situation on onlineteaching and learning, it is necessary to describe the language style that remained dominant throughout the situation. Zhao (2020) in his blog. 'What's Coronavirus doing to our language?' reported that our language is also being affected by Coronavirus. There is new vocabulary, plenty of terminologies, and multilingualism growing. The transition to online teaching and homeschooling is also delicate and diverse. Many linguists have examined these changes and variations in language, but the current research has a much broader perspective as it is aimed at observing the language style of popular social media, Twitter. Secondly, by using the comprehensive methodology of 
multidimensional analysis which is the most reliable framework to observe maximum linguistic variation in language, genre, register, or variety. Thirdly, the data/corpus is compiled largest to determine the language style of tweets.

$\mathrm{Hu}$, Talmadupula, \&Kabbanpati(2013) has already conducted research to determine the language style of Twitter. In the study, it was inferred that the content sharing mechanism and language style of Twitter is debatable. Regarding size limitations, it is nearer to chat and SMS, and the communication content is related to emails, blogs, and newspapers. In this research, by applying the analytical research methodology of gathering a particular set of linguistic features and flexible factorization, the tweeter language style is determined on four grounds. The language style is more conservative on Twitter, and it is less formal as compared to SMS and online chat. Twitter users have unique language styles most of the time. The temporal references in Twitter appear to be more like SMS and chat. Twitter has less linguistic variation than other formal media mediums.

In another study on the same arguments, Clarke (2019) determined major dimensions of linguistic variation named as interaction vs. non-interaction and 'challenging vs. non-challenging. Fundamentally, the research explored trolling, a behavioral pattern in Tweets by applying the multidimensional (MDA, 1988) approach. It revealed that identical to informal communication, Twitter's language nature is more interactive. According to the second functional dimension in this study Tweeter trolls employ more covert strategies. The challenging functional dimension showed the hostile nature of tweet users.

Consequently, in the light of the above-mentioned studies, Twitter's language displays a continuous range of linguistic variation.

\section{Material and Methods}

\section{Target Corpus Description and Compilation}

Keeping in view the objectives of the research, i.e., to examine the major cooccurring linguistic features, determine language style of tweets and find out distinguishing features, the largest Tweets corpus is started to build up. Burghardt (2015) purposes existing tools to collect and analyze Twitter data and to conduct a study on new media. It is described that some API tools, for example, Twista (Spanner et al, 2015), Tworpus (Bazo et al, 2013), and Tweet Archivist (Williams, et al, 2011) to save, export and visualize the Tweets data/corpus. These tools are categorized into 'streaming API tools' and 'search API tools. The latter category and tool: Tweet Archivistis carried out to download the collection of tweets for the present research. While compiling the corpus, firstly the access to the tool was achieved by requesting the owner through the website https://www.tweetarchivist.com/ . These services can be allowed to the researchers for academic purposes. There is a time limit for access and the tool runs the standard tier of the Twitter Search API to poll Twitter on the researcher's behalf. The 
researcher archive based on a particular key term (as in the current study the key term was 'Online Education') grows to maximum tweets in with time-bound and data gathered. Secondly, with the permission of the owner, the data is exported in Pdf form of the text file and ready corpus.

\section{Target Corpus Nature}

The nature of the corpus is real 'world' text that is used for empirical investigations of language use, style, and variation. When the tool is launched, it pulls maximum results with automatic configuration of relevant tweets based on the key term in the textbox. It allows visualizing maximum tweets, top users, and language breakdown. Through language breakdown, top words are determined with frequency.

Consequently, the data of 0.2 million tweets consisting of 4,996,456 words in total is compiled and these tweets cover 32 days, i.e., from July 7, 2020, to August 8, 2020.

It is also necessary to describe the statistical situation of the pandemic during the above-mentioned time limit. Therefore, the following figure can help us graphically understand the COVID-19 situation all over the world at that time.

\section{Figure 1}

An Overview of the Covid-19 Worldwide Situation

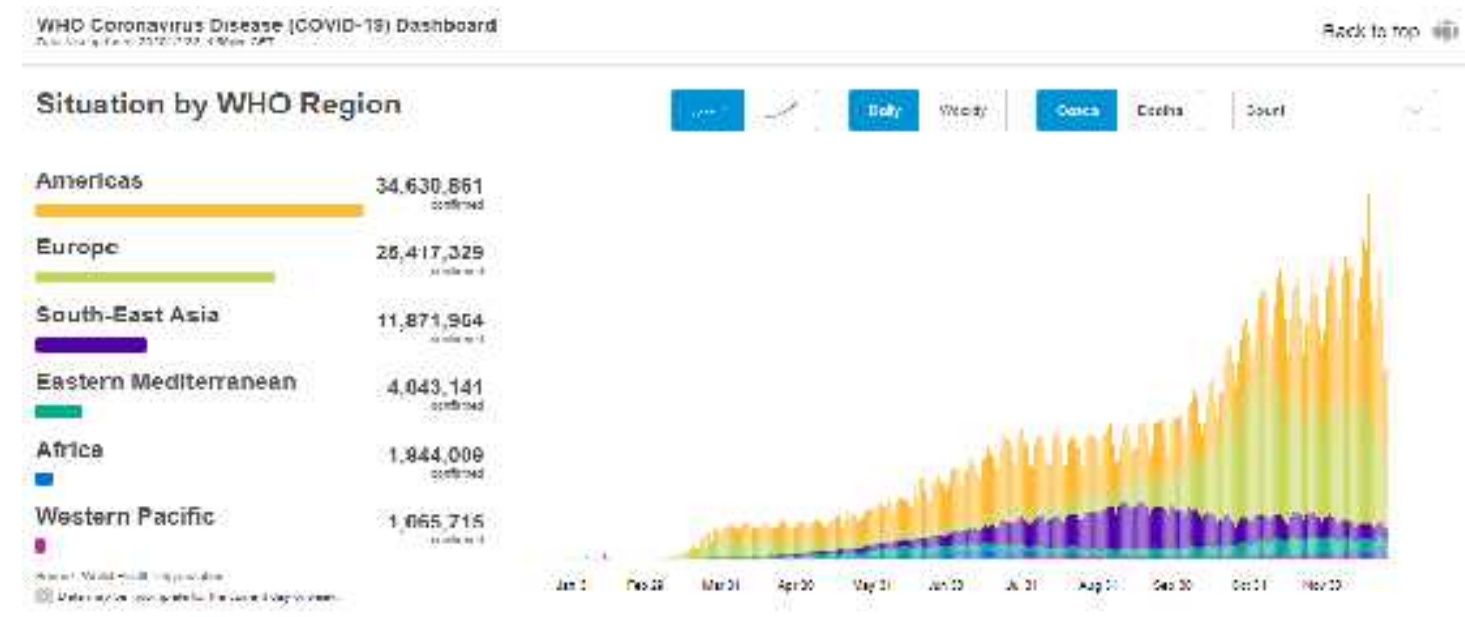

The graphical representation has been obtained from the official website of the World Health Organization (WHO) (https://covid19.who.int/ ) on December28, 2020, to get the latest statistics on the pandemic. According to figure 1 , the novel coronavirus out broke around March, got its peak in June 2020, the mortality rate, confirmed case, and tests performed started raising in July and August and got an increased number. Later, all the months witness the rise in the 
COVID-19 cases since then. Globally, there are more than 1.2 million mortalities while more than fifty-one million have been affected by the virus.

The detailed statistics about the source of tweets, volume over time, users' mention, and influencer index are provided in the Appendix.

Equally, Fig 2 presents Covid-19's staggering impact on the education sector.

Figure 2

An Overview of global education during Covid-19

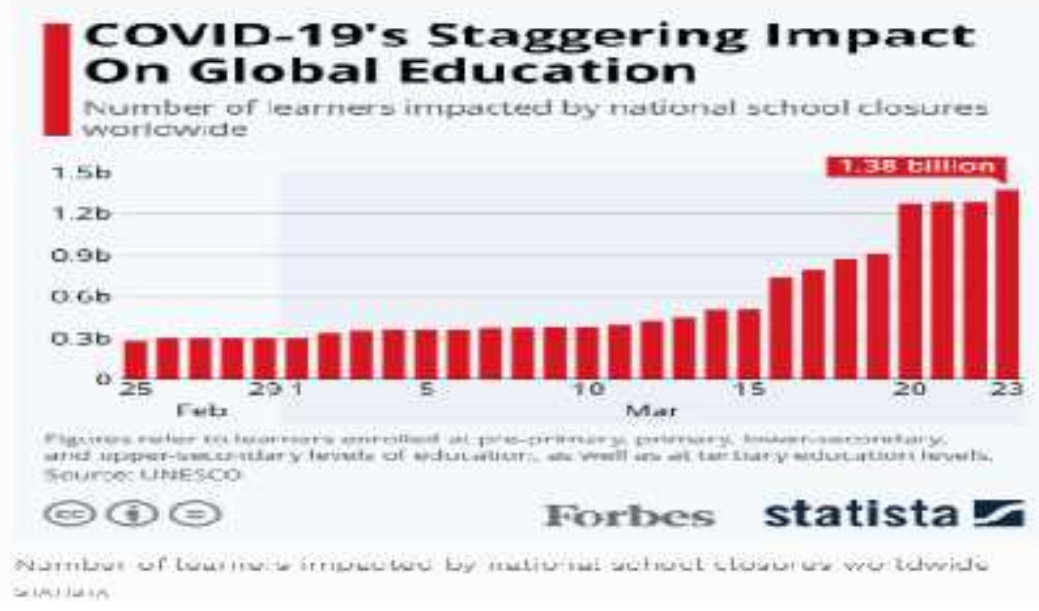

The graphical representation has been obtained from the official website of the United Nations Educational, Scientific, and Cultural Organization (UNESCO)( https:/ / en.unesco.org/ ) According to the Forbes report of UNESCO data, above 1.3 billion learners worldwide discontinued to attend schools or universities since March 2020. Primary, secondary, and tertiary levels of education got closure and $1,379,344,914$ students who are $80 \%$ of total global enrolled students were unable to attend their institutions. It resulted in an unprecedented challenge to develop a longdistance education system.

\section{Data Analysis}

According to Biber \& Conrad (2009) to study the different styles adopted by speakers, the best way is to retrieve the most frequent and ubiquitous forms. Therefore, the first quantitative linguistic step is initiated by categorizing the most occurred words. For this purpose, the lists of the top words in tweets are autogenerated using the computational tool Tweet Archivist (www.tweetarchivist.com). This allowed us to see the parts of speech commonly used by speakers. Later, MAT tagger (explained thoroughly below) is used to determine the set of linguistic feature occurrences to define the language style. Table 1 consists of the top 25 English words used in tweets during the pandemic months. 
Table 1

Top 25 Words in Tweets Corpus

\begin{tabular}{cccccccc}
\hline $\begin{array}{c}\text { Sr. } \\
\text { No. }\end{array}$ & Top Words & Count & POS & $\begin{array}{c}\text { Sr. } \\
\text { No. }\end{array}$ & Top Words & Count & POS \\
\hline 1 & Online & 8,630 & JJ & 14 & Their & 2,916 & PRP\$ \\
\hline 2 & Can't & 8,508 & VBP & 15 & Reopen & 2,904 & VB \\
\hline 3 & Expect & 8,440 & VBP & 16 & Secretary & 2,795 & NN \\
\hline 4 & Students & 8,309 & NNS & 17 & Going & 2,285 & VBG \\
\hline 5 & Schools & 5,990 & NNS & 18 & Board & 2,014 & NN \\
\hline 6 & Learn & 5,340 & VB & 19 & Access & 2.009 & NN \\
\hline 7 & National & 4,662 & JJ & 20 & Fall & 1,814 & NN \\
\hline 8 & Asked & 4,540 & VBN & 21 & Pandemic & 1,751 & JJ \\
\hline 9 & Anthems & 4,481 & NNS & 22 & Teachers & 1,437 & NNS \\
\hline 10 & Exams & 3,637 & NNS & 23 & Universities & 1,326 & NNS \\
\hline 11 & Classes & 3,589 & NNS & 24 & Start & 1,289 & VB \\
\hline 12 & Learning & 3,222 & NN & 25 & International & 1,261 & JJ \\
\hline 13 & Public & 3,032 & JJ & & & & \\
\hline
\end{tabular}

$\mathrm{JJ}=$ Adjective

$\mathrm{VBP}=$ Verb, non-3 ${ }^{\text {rd }}$ person singular present

VBN= Past tense or irregular past participial verb

NNS $=$ Noun, plural

$\mathrm{NN}=$ Noun, singular, or mass

PRPS $=$ Possessive pronoun

$\mathrm{VBG}=$ Verb, gerund or present participial

The table shows an intensive use of seven verbs (Can't, expect, learn, asked, reopen, going, and start) and eleven nouns (students, schools, anthems, exams, classes, learning, secretary, board, access, teachers, and universities) and five adjectives (online, national, public, pandemic and international). Interestingly, one pronoun 'their' is also occurring frequently. Most frequent words/parts of speech just provide a general description of individual linguistic features. As to address the present research problem, it is required to observe the style/register/text type of the target tweet data, therefore multidimensional analysis is conducted below through MAT tagger.

MAT Tagger (The Multidimensional Analysis Tagger)

It is a computer application to analyze the corpus while using the multidimensional framework developed by Biber (1988). Through Stanford tagging, input data and statistical counting of the sets of linguistic features which co-occur then finally matching these values with the same framework reference values. The 
original reference values are indeed based on LOB corpus (Lancaster-Oslo-Bergen Corpus, millions word collection of British English, compiled in 1970). Consequently, the target data's text style/type and other grammatical and functional information are obtained. MAT tagger replicates completely Biber's (1988) framework's functional dimension scores and text types. It can be generalized and applied to other data sets due to its testing reliability (Nini, 2019). In simple words, the automatic computational tool provides a facility that the mean values of any data set are compared to British texts, and the language style or text type of that data set is defined.

The use of MAT tagger on target data of tweets is of particular significance for methodological rationale due to the following reasons:

a) It is a complete computational tool for corpus analysis with grammatical tagging and statistical counting of 67 linguistic features and their co-occurrence (set of linguistic features) to determine text type or style of language.

b) For 30 years back, based on Biber's (1988) framework, the tagger is being vastly used to determine academic language style (e.g., Biber 2003; Gray 2013), to examine web registers/vocabulary (Grieve, Biber, and Friginal, 2011; Biber \& Egbert 2016), to find author styles (Finegun, 1994), to observe regional language variation (Grieve, 2014; Biber \& Burges, 2000) and for many other language styles determining studies.

c) After tagging and statistical analysis (mean and standard deviation) of linguistic features and their co-occurrence, the tagger provides six functional/thematic dimensions of the target corpus. It determines the language style of the audience.

These are:

1. Involved vs. informational discourse (communication)

2. Narrative vs. non-narrative concerns

3. Context independent vs. context-dependent discourse

4. Overt expression of persuasion/ Argumentative text

5. Abstract vs. non-abstract information

6. Online informational elaboration

With these six functional dimensions of text Biber (1989) has also found eight text types such as:

1. Intimate interpersonal interaction 
2. Informational interaction

3. Scientific exposition

4. Learned exposition.

5. Imaginative narrative

6. General narrative exposition

7. Situated reportage

8. Involved persuasion

\section{Results and Discussion}

Interestingly, tagger determines these functional or meaning dimensions on covariations of linguistic features which are determined by mean values. Later. High or low mean score categorized the text dimension profile.

For example, according to the Biber (1988) framework, and MAT tagger replicates that context if the private verbs such as in the current research tweet data, 'can't', 'expect', and present tense forms such as 'reopen', 'going' and 'start' are frequently found hence it shows the style of communication/text as interactive or involved. Also, private verbs are verbs to express thoughts, intellectual ability of emotions (Oxford dictionary) (www.oxfordreference.com ).

The clustering of all linguistic features based on the dimensions mean scores are given in fig. 3 below. Moreover, the above-mentioned reasons to choose MAT tagger analysis for present research ensure the validity of the tool and reliability of results.

\begin{tabular}{|c|c|c|c|c|c|c|c|}
\hline \multicolumn{8}{|c|}{$\begin{array}{l}\text { Comparison between Mean Dimensional Scores of Tweet } \\
\text { Corpus \& LOB Corpus }\end{array}$} \\
\hline \multirow{2}{*}{$\begin{aligned} & 10 \\
- & -28 \\
- & -30\end{aligned}$} & $\square$ & - & - & 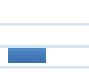 & $-=$ & \multicolumn{2}{|c|}{-} \\
\hline & D1 & D2 & D3 & D4 & D5 & D6 & $\begin{array}{l}\text { Text } \\
\text { Type }\end{array}$ \\
\hline - Tweet_Corpus Score & -22.61 & -6.03 & 2.87 & -7.54 & -3.29 & -2.41 & 0 \\
\hline LOB_Corpus Score & -14.9 & -2.6 & 4.2 & -0.5 & 5.5 & -1.6 & 0 \\
\hline \multicolumn{8}{|c|}{ - Tweet_Corpus Score } \\
\hline
\end{tabular}

Figure 3 Comparison Between Mean Dimensional Scoresof Tweet Corpus \& LOB $\mathrm{D}=$ Dimension

\section{Corpus}


After running the MAT tagger on tweet corpus, there come interesting findings based on dimensions mean scores, the language used in tweets is involved, non-narrative, context-dependent, and non-argumentative.

Overall, the text type or functional dimension of data is 'learned exposition'. In-text linguistics learned exposition contains official documents, academic prose, and press reviews, etc. tagger results proved this stance through the lowest score on D1 and D2, a high score on D3, and low scores on D4 and D6. Only the D5 lowest scores, contrary to LOB corpus provides a different function of the text even from learned exposition text type. It represents the tweet text as intimate interpersonal interaction and informational interaction which is generally not considered the characteristic of learned exposition. According to Nini (2019), it determines that on this dimension the text style is not formal like official documents or academic prose rather it resembles an interaction between personal friends. Comparing all dimensions mean scores with LOB corpus or British texts of a particular register of learned exposition, some similarities, and differences in values are found. Mentioning the D1 score, the text type of learned exposition in the LOB corpus has a low score and is considered also as involved rather than informational. But in the tweet corpus, the results show the nature of the corpus as more involved (functional dimension) than the LOB corpus. D2 score demonstrates that although both corpora are non-narrative and tweet corpus seems to have this functional meaning to a greater extent. The results of D3 show slight variation among tweet corpus and LOB corpus and both are context-dependent in this regard. Surprisingly, the D4 results are striking as in LOB corpus, learned exposition mean values demonstrate that this text type is slightly non-argumentative, but tweet corpus broke through that tweet users are extremely non-argumentative. The lowest score on this dimension changes the text type from learned exposition to situated reportage. This text type usually includes online commentaries of the events in progress. Likewise, D5 mean scores of tweet corpus are also extraordinarily opposite from LOB corpus. It also changes the style of text from learned exposition to intimate interpersonal interaction and informational interaction. D6 score while represents nearly similar scores.

Now, it is necessary to describe the clustering of linguistic features with their grammatical information on all these dimensions of D1, D2, D3, D4, D5, and D6. It overall determines the text type of tweet corpus majorly as learned exposition and according to D4 score non-argumentative greatly and concerning D5 score as intimate interpersonal interaction and informational interaction. On dimension 1, informational vs. involved, the tweet target has represented the highly involved text type according to MAT tagger results.Hu, Y., Talmadupula, K., Kabbanpati, S. (2013) already conducted research to determine the language style of Twitter. In the study, it was inferred that the content sharing mechanism and language style of Twitter is debatable. Concerning size limitations, it is nearer to chat and SMS, and the communication content is related to emails, blogs, and newspapers. The language style is more conservative on Twitter and it is less formal as compared to SMS and online chat. The present research is also being supported by this result. 
It demonstrates that the tweet corpus has contained, loosely joined clauses, the density of 1st person pronouns, emphatics, hedges, and excessive usage of pronoun 'it'. (Reppen, Fitzmauice\& Biber 2002). According to the multidimensional framework of Biber (1995), if on the scale of D2, text type proves as involved rather than informational, then there must be fragmented phrases and generalized content found in the text. On the scale of dimension 2, there is high loading of the clustering of present tense verbs, attributive adjectives, and private verbs that is also presented in table 1, above when top frequent individual parts of speech are automatically derived. It produces the text functional dimension as nonnarrative (Biber \& Conrad, 2019). Dimension 3 i.e., context-free text vs. contextdependent results of tweet corpus shows the language structure as contextdependent that elaborates the presence of concrete spoken style and simple structure of phrases and clauses in target data of tweets (Biber, 1995). Along with the results of dimension 4, the tweet corpus is found as highly non-argumentative which means that there is the absence of a personal stance on an issue in the text and simply the users have provided the information (Biber, 1994). Lastly, the dimension 5 results show a non-impersonal style of writing reflecting more explanation and exemplification of an issue present in the text. (Biber \& Conrad, 2019).

\section{Conclusion}

Overall results of multidimensional analysis of tweet corpus characterize this text type as 'learned exposition'. These text types are usually composed of informational expositions by focusing on facts and figures presentation, message delivery, and particular findings. Biber's(1994) framework of multidimensional analysis of the text characterized that 'learned exposition' is found in official documents, academic prose, and press reviews. Mentioning the D1 score, the text type of learned exposition in the LOB corpus has a low score and is considered also as involved rather than informational. But in the tweet corpus, the results show the nature of the corpus as more involved (functional dimension) than the LOB corpus. D2 score demonstrates that although both corpora are non-narrative and tweet corpus seems to a greater extent. The results of D3 show slight variation among tweet corpus and LOB corpus and both are context-dependent in this regard. Surprisingly, the D4 results are striking as in LOB corpus, learned exposition mean values demonstrate that this text type is slightly non-argumentative, but tweet corpus broke through that tweet users are extremely non-argumentative. The lowest score on this dimension changes the text type from learned exposition to situated reportage. This text type usually includes online commentaries of the events in progress. Likewise, D5 mean scores of tweet corpus are also extraordinarily opposite from LOB corpus. It also changes the style of text from learned exposition to intimate interpersonal interaction and informational interaction. D6 score while represents nearly similar scores. The background of tweet users might be native or nonnative. As Arguelles \& Munoz (2013) found in a Tweet corpus research that general discourse and organized features are common in native and non-native tweets.Moreover, the content sharing mechanism and language style of Twitter is debatable (Hu, Talmadupula\&Kabbanpati, 2013). 
Consequently, presuming distinctive linguistic features, the style and structure of the Tweeter language are meant for conveying information about any issue. The linguistic variation is combined with an intimate/interpersonal manner. 


\section{References}

Abbasi, S. Ayoob, T. Malik, A. \& Memon, S.I. (2020). 'Perceptions of Students Regarding E-Learning during Covid-19 at a Private Medical College'. Pakistan Journal of Medical Sciences, vol. 36, no. COVID19-S4,

Patricia Aguilera-Hermida, A. (2020) 'College Students' Use and Acceptance of Emergency Online Learning Due to COVID-19'. International Journal of Educational Research Open, vol. 1, 100011.

Argüelles Álvarez, Irina, \& Alfonso Muñoz Muñoz. (2012).'An Insight Into Twitter: A Corpus Based'. Revista de Lingüística y LenguasAplicadas, vol. 7, no. 1, 37-50.

Bao, W. (2020). Covid-19 and Online Teaching in Higher Education A Case Study of Peking University.WileyPreodicals LLC, Pg. 113-115

Bazo et al, (2013).Tworpus- An easy-to-use tool for the creation of Tailored Tweeter Corpora. In I. Gurevych et al (Eds.), Proceedings of the 25 th International Conference of the German Society for Computational Linguistics and Language Technology, GSCL' 13 (pp 23-34) Heidelberg: Springer

Biber, D \& Burges, J. (2000). Historical change in the language use of Women ad Men. Gender Differences in Dramatic Dialogue.Journal of English Linguistics, 28 (1), 21-33.

Biber, Douglas, \& Susan Conrad (2009). Register, Genre, and Style. Cambridge University Press

Biber, D (1988).Variation Across Speech and Writing. Cambridge University Press Cambridge

Biber, D. \& Egbert, J. (2016). Using Multi-Dimensional analysis to study register variation on the searchable web. Corpus Linguistic Research, (2) 1-23.

Biber, Douglas, \& Edward Finegan (1994). Sociolinguistic Perspectives on Register. Oxford University Press

Biber, Douglas, \& Federica Barbieri (2007). 'Lexical Bundles in University Spoken and Written Registers'. English for Specific Purposes, vol. 26, no. 3, 263-86.

Burghardt, M. (2015). Introduction to Tools and Methods for the Analysis of Twitter Data. Living Linguistics, (1)volume 1 and issue 1, 74-91

Clain, M., Gibbs, K \& McRae, B (2020). “Please Explain!"-Public Perception of Students with Diversity in Mainstream Education as Voiced in Australian Online News Media'. International Journal of Educational Research Open, vol. 1, 100006. 
Clark, I. (2019). Functional Linguistic Variation in Twitter Trolling, The International Journal of Speech, Language, and the Law. 26 (1).

Finegun, E. (1994).Sociolinguistic Perspectives on Register, Oxford Studies in Sociolinguistics. Oxford University Press New York.

Biber, Douglas, \& Federica Barbieri (2007). 'Lexical Bundles in University Spoken and Written Registers'. English for Specific Purposes, vol. 26, no. 3, 263-86.

Biber, Douglas, \& Bethany Gray (2013). ‘Discourse Characteristics Of Writing And Speaking Task Types On The Toefl Ibt ${ }^{\circledR}$ Test: A Lexico-Grammatical Analysis'. ETS Research Report Series, vol. 2013, no. 1, 128.

Tyagi, Priyanka, and R. C. Tripathi. (2019). 'A Review towards the Sentiment Analysis Techniques for the Analysis of Twitter Data'. SSRN Electronic Journal, DOI.org (Crossref), https://doi.org/10.2139/ssrn.3349569.

Grieve, J. (2014).A Multidimensional Analysis of regional variation in American English, Multi-Dimensional Analysis, 25 years on A tribute to Douglas Biber.John Benjamins Publishing Company 60

Grieve, J., Biber, D. \& Friginal, E. (2011). Variation among blogs: A multidimensional analysis. Genres on the Web, 42, 303-322.

Hu, Yuheng, (2013). 'Dude, Srsly?: The Surprisingly Formal Nature of Twitter's Language'. Proceedings of the 7th International Conference on Weblogs and Social Media, ICWSM 244-53.

McCarthy, N. (2020). UNESCO: COVID-19 School Closures Have Impacted Nearly 1.4 Billion Students [Infographic] Forbes 24/03/2020

Mishra, Gupta, and Shree (2020).Online Teaching-Learning in Higher Education during Lockdown Period of COVID-19 Pandemic'. International Journal of Educational Research Open, vol. 1, 100012

Nini, A. (2019). Introduction to Forensic Linguistics. Guest lecture at the University of Bamberg, Bamberg, Germany. 10/12/2019.

Nini, A. (2019). The Multi-Dimensional Analysis Tagger. In Berber Sardinha, T. \&Veirano Pinto M. (eds), Multi-Dimensional Analysis: Research Methods and Current Issues, 67-94.

Oyedotun, T. D (2020). 'Sudden Change of Pedagogy in Education Driven by COVID-19: Perspectives and Evaluation from a Developing Country'. Research in Globalization, vol. 2, 100029. 
Reppen, R. Fitzmauice, S \& Biber, D (2002).Using Corpora to Explore Linguistic Variation. John Benjamins Publishing

Rogers, R (2013). Debanalizing Twitter. The Transformation of an Object of Study. In Proc. $5^{\text {th }}$ Annual ACM Web Science Conference (pp 356-365)

Shehzad, S.K (2020). Impact of Virtual Teaching on ESL Learners Attitudes under Covid-19 Circumstances at Post Graduate level in Pakistan, English Language Teaching.Canadian Center of Science and Education, 13 (9), 1-9.

Slimi, Zouhaier. (2020). 'Online Learning and Teaching during COVID-19: A Case Study from Oman'. International Journal of Information Technology and Language Studies, vol. 4 , no. 2

Spanner, S. (2015). Twista- An Application for the Analysis and Visualization of Tailored Tweet Collections. In Proceed. Of the $14^{\text {th }}$ International Symposium of Information Science (pp 191-202).

Zhao, H. (2020). Multilingualism: Empowering Individuals, Transforming Societies (MEITS). https://www.meits.org/blog/post/whats-coronavirus-doing-to-ourlanguages. 